\title{
Topographic Imaging of Quantitative EEG in Response to Smoked Cocaine Self-Administration in Humans
}

\author{
Malcolm S Reid*,', Frank Flammino', Bryant Howard ${ }^{1,2}$, Diana Nilsen ${ }^{3}$ and Leslie S Prichep ${ }^{1,2}$ \\ 'Department of Psychiatry, New York University School of Medicine, New York, NY, USA; ${ }^{2}$ Brain Research Laboratories, New York University \\ School of Medicine, New York, NY, USA; ${ }^{3}$ Department of Pulmonary Medicine, New York University School of Medicine, New York, NY, USA
}

\begin{abstract}
Quantitative electroencephalographic (qEEG) profiles were studied in cocaine-dependent patients in response to an acute, single-blind, self-administered dose of smoked cocaine base $(50 \mathrm{mg})$ vs placebo. qEEG data were averaged using neurometric analytical methods and the spectral power of each primary bandwidth was computed and topographically imaged. Additional measures included cocaineinduced high, craving, and related subjective ratings, heart rate, blood pressure, and plasma cortisol and homovanillic acid levels. In all, I 3 crack cocaine-dependent subjects were tested. Cocaine produced a rapid increase in subjective ratings of cocaine high and good drug effect, and a more persistent increase in cocaine craving and nervousness. Cocaine also produced a rapid rise in heart rate and a prolonged increase in plasma cortisol. Placebo, administered in the context of cocaine cues and dosing expectations, had no cocaine-like subjective or physiological effects. Cocaine produced a rapid increase in absolute theta, alpha, and beta power over the prefrontal cortex (FPI, FP2), lasting up to 25 min after dosing. The increase in theta power was correlated with good drug effect, and the increase in alpha power was correlated with nervousness. Cocaine also produced a similar increase in delta coherence over the prefrontal cortex, which was positively correlated with plasma cortisol, and negatively correlated with nervousness. Placebo resulted in an increase in alpha power over the prefrontal cortex. These data demonstrate the involvement of prefrontal cortex in the qEEG response to acute cocaine. Evidence indicates slow wave qEEG, delta and theta activity, involvement in the rewarding properties of cocaine. Neuropsychopharmacology (2006) 31, 872-884. doi: I0. I038/sj.npp. I 300888; published online 28 September 2005
\end{abstract}

Keywords: cocaine; cues; cortisol; electroencephalography; craving; reward

\section{INTRODUCTION}

In recent years, neuroimaging studies have extensively characterized the acute neurobiological effects of cocaine in cocaine-dependent patients. These studies have identified numerous brain regions activated by cocaine, including the basal forebrain, ventral tegmentum, caudate, nucleus accumbens, thalamus, cingulate, and prefrontal cortex (Breiter et al, 1997). Further characterization of the acute effects of cocaine in humans has revealed dose- and timedependent reductions in cerebral blood volume (Kaufman et al, 1998a, b), cerebral blood flow (Wallace et al, 1996), and in glucose metabolism (Bartzokis et al, 1999). In some studies, a correlation between the subjective rewarding effects of cocaine and the alteration of neuronal metabolism and cerebral blood volume was shown (Breiter et al, 1997; Kaufman et al, 1998a,b), indicating the involvement of mesolimbic regions in the rewarding effects of cocaine.

*Correspondence: Dr MS Reid, Department of Psychiatry, New York University School of Medicine, VANYHHS, I I 6A, 423 East 23rd Street, New York, NY I00I0, USA, Tel: + I 212686 7500x7983, Fax: + I 212951 6891, E-mail: malcolm.reid@med.va.gov

Received 5 May 2005; revised 19 July 2005; accepted I August 2005 Online publication: 4 August 2005 at http://www.acnp.org/citations/ Npp080405050296/default.pdf
Prior to these neuroimaging studies, investigations with electroencephalographic (EEG) measurements provided some of the first in vivo data on the acute effects of cocaine in the human brain. Berger (1931), the first to study the effects of cocaine on human EEG, reported an increase in activity in the beta bandwidth. This was replicated in subsequent studies with a larger number of subjects by Herning et al $(1985,1994)$, who found a correlation between enhanced beta levels and plasma cocaine levels. Interestingly, they have also reported an increase in delta activity (Herning et al, 1985) and frontal alpha activity (Herning et al, 1994), while others have reported an increase in alpha wave EEG associated with bursts of cocaine-induced euphoria (Lukas et al, 1990; Lukas, 1991). None of these studies, however, were able to map the location of the EEG effect to determine if the effects were neuroanatomically specific.

More recently, we have begun analyzing the quantitative electroencephalographic ( $\mathrm{qEEG}$ ) profiles of cocaine-dependent patients using neurometric analytical methods to topographically image the spectral power of each primary bandwidth over the cortical surface. Our group, and others, have reported excess alpha activity (Alper et al, 1990; Lukas, 1991; Herning et al, 1994; Roemer et al, 1995; Prichep et al, 1996a) and decreased delta activity (Alper et al, 1990; Noldy 
et al, 1994; Roemer et al, 1995; Prichep et al, 1996a, b), while others have reported increased beta power (Herning et al, 1985, 1997; Noldy et al, 1994) in cocaine-dependent patients, recorded in eyes closed, resting conditions. The qEEG abnormalities, primarily found in anterior cortical regions, were shown to correlate with the amount of prior cocaine use (Prichep et al, 1996a; Herning et al, 1997; Roemer et al, 1995). In a recent cue exposure study, we found evidence for an increase in beta power during the handling of cocaine paraphernalia and the viewing of a crack cocaine video, and an increase in delta power during guided imagery related to cocaine craving. In both cue procedures, the primary qEEG changes were over the prefrontal cortex (Reid et al, 2003). These findings demonstrate the ability to neuroanatomically map qEEG profiles at rest and during psychological stimulation in cocaine-dependent patients. Moreover, the increase in beta and delta activity during cocaine cue exposure is consistent with previous EEG studies on the acute effects of cocaine (Berger, 1931; Herning et al, 1985, 1994). As a follow-up to these studies, we have investigated qEEG activation profiles induced by an acute dose of smoked cocaine base (50 mg), $v s$ placebo, in cocaine-dependent patients. Concurrent with the collection of qEEG data, we assessed the subjective, neurendocrine, and physiological effects of cocaine and compared these effects with the qEEG profiles.

\section{METHODS}

This study was approved by the New York University School of Medicine Institutional Review Board and performed under FDA approved IND (\# 60,631). Cocaine patient recruitment, screening, and testing took place at the New York University School of Medicine General Clinical Research Center (NYU GCRC) outpatient facilities and the New York University Medical Center, Tisch Hospital Inpatient Psychiatry Clinic. All subjects provided informed written consent to participate.

\section{Subjects}

Participating subjects were crack cocaine dependent, with active habits verified by a urine drug screen positive for cocaine in the prior 2 weeks, and were not seeking or enrolled treatment for cocaine abuse. Subjects were excluded if they had co-morbid dependence on alcohol or other drugs of abuse (except nicotine), were using prescribed psychotropic medications, or were diagnosed with an axis I psychiatric disorder according to DSM-IV criteria from the SCID (other than addiction) requiring treatment. A total of 13 patients were tested. The group consisted of 11 males and two females, average age $38.6 \pm 11.0$, with ethnicities: one Hispanic, three Caucasians, and nine African-Americans. Nine of 13 subjects were cigarette smokers and alcohol use (lifetime use any: $17.2 \pm 4.9$ years; use over last 30 days: $6.3 \pm 1.8$ days) indicated moderate levels of drinking behavior. Cocaine use over the lifetime $(12.8 \pm 3.7$ years $)$ and over the last 30 days $(12.5 \pm 3.6$ days) indicated chronic history of abuse. Subjects reported spending $\$ 110 \pm 31$ on cocaine during the week prior to hospital admission and the number of days since last use, prior to the test day, was brief $(3.1 \pm 0.9)$.

Subjects meeting the study eligibility criteria were admitted into the Psychiatry Inpatient Clinic where they remained hospitalized as inpatients for $24 \mathrm{~h}$ prior to testing and for overnight observation following testing. Baseline evaluations during the pre-testing 'washout' day included: Drug Use Survey (last 30 days), Brief Cocaine Craving Scale (BSCS) (Mezinskis et al, 1998), and the Addiction Severity Index (ASI) (McLellan et al, 1985). During the 24-h inpatient hospitalization, subjects were allowed to smoke cigarettes 2-3 times a day under staff escort and standard hospital meals were provided. On the morning of testing the patients had a low-fat breakfast, followed by a cigarette break (for those that were smokers) approximately $1 \mathrm{~h}$ before beginning test procedures.

\section{Cocaine Smoking Tests Procedures}

The cocaine base smoking procedures took approximately $6 \mathrm{~h}$, including setup and removal of intravenous (i.v.) catheters, physiological monitoring equipment, and the EEG electrodes. Testing employed a within-session, crossover study design, in which each subject self-administered a cocaine dose (50 mg, cocaine base formula) followed $3 \mathrm{~h}$ later by a placebo dose (or vice versa). The order of dosing was randomly selected on a $1: 1$ ratio, using block sizes of 4 , and was administered in a single-blind fashion. The cocaine base and placebo smoking sessions were designed based on previous cocaine self-administration studies of Foltin and colleagues (Foltin and Fischman, 1991; Foltin and Haney, 2000). All patients had at least one practice session with a blindfold and empty stem prior to testing. All patients were informed that during each test session they had a 50:50 chance of receiving the $50 \mathrm{mg}$ dose.

Subjects smoked from a standardized glass stem, a commonly used form of crack cocaine smoking paraphernalia in the New York City metropolitan area. The stems were made from glass Pyrex tubes ( $8 \mathrm{~mm}$ diameter) (Fischer Scientific) cut into $10 \mathrm{~cm}$ lengths, with a metal screen placed approximately $1 \mathrm{~cm}$ from one end. In order to obtain oral stimulation (smell and taste) similar to that of a used stem, three $10 \mathrm{mg}$ cocaine base pellets had been burned through the tubes using a lighter and vacuum line under a ventilated laboratory hood. Separate 'pre-used' stems were included in the cocaine $(50 \mathrm{mg}$ ) and placebo $(0 \mathrm{mg})$ smoking conditions, and were replaced after every four subjects.

Upon the beginning of testing, a standard automated blood pressure cuff and an 18-gauge catheter was inserted into a subcutaneous vein (for repeated blood draws, kept patent by infusion of a dextrose $(5 \%)$ solution at $100 \mathrm{~cm}^{3} / \mathrm{h}$ ) on the patient's non-writing arm, ECG and heart rate monitor leads were applied to the chest, and EEG leads were placed on the scalp. Initially, blood samples were taken, the patient was given the Cocaine Dose Rating Scale and the Mood Analog Scale, and then $10 \mathrm{~min}$ ( 5 min eyes closed, 5 min eyes open) of baseline resting EEG, blood pressure and heart rate were collected. Thereafter, each individual underwent a 1-2-min cocaine cue exposure procedure that involved briefly viewing crack cocaine-related items (previously used glass stems, $\$ 20$ bill, lighter, small plastic 'dime bags' containing simulated crack) placed in front of him/her 
on a table top, being instructed that they 'are now going to smoke a dose of crack cocaine', and selecting a glass stem from which to smoke crack cocaine. This procedure was designed to replicate the conditioned stimuli that patients normally experience prior to smoking crack cocaine (CS + ). To that degree, all enrolled subjects had reported that their standard route of administration was smoking via a glass stem, and all tested subjects reported that their selected stem was similar to those which they normally use.

Following the pre-dosing cue procedure, heart rate and blood pressure were assessed to assure that cardiovascular activity was not above criteria for safe drug administration $(\mathrm{HR}<130, \mathrm{DP}<100, \mathrm{SP}<165)$, and then the cocaine or placebo smoking session began. With the cocaine cues in view in front of the patient, the research staff member (same individual was involved in all sessions) placed a blind-fold over the patient's eyes, placed a cocaine dose $(50 \mathrm{mg})$ or nothing (placebo condition) in a glass stem, and then handed the glass stem back to the patient. The actual stem used for smoking was a standardized stem specifically prepared for the study, not that selected by the patient. Using a lighter, the staff member held the flame over the cocaine dose and the subject was instructed to take one large inhalation and hold it as long as they would under routine smoking practices. Once the patient had inhaled the dose and then exhaled, the staff member took the stem from the patient, placed it out of view from the patient, and removed the blindfold. The cocaine cues from prior to dosing remained in view in front of the patient. ECG was monitored continuously, and heart rate and blood pressure were assessed every $5 \mathrm{~min}$, beginning $30 \mathrm{~min}$ prior to cocaine dosing and continuing for $1 \mathrm{~h}$ following dosing (every $2.5 \mathrm{~min}$ for $20 \mathrm{~min}$ following dosing). The Cocaine Dose Rating Scale and the Mood Analog Scale were completed at 5, 10, 20, 40, and $60 \mathrm{~min}$ post-dose administration, followed by blood samples collected at the same time points (except for the $5 \mathrm{~min}$ time point). The physiological and EEG recordings were recorded continuously throughout the session. Eyes open, resting EEG data were obtained during minutes $0-5,15-20,35-40$, and $55-60$ post-dose administration, and eyes closed, resting EEG were obtained during minutes $5-10,20-25,40-45$, and $60-65$ post-dose administration.

Following completion of the day's testing procedures, vital signs and psychiatric status were monitored by clinical staff until all vital signs returned to pre-testing levels. In addition, patients underwent overnight clinical observation by the NYU GCRC and Tisch Hospital Psychiatry Inpatient Clinic nursing staff prior to discharge.

\section{Assessments}

Cocaine high and related subjective effects were assessed using the Cocaine Session Rating Scale, based on the work of Foltin and colleagues (Foltin and Fischman, 1991; Foltin and Haney, 2000) and our previous cocaine-craving studies (Reid et al, 2003), while mood variables were assessed using the Mood Analog Scale (Berger et al, 1996).

Cocaine session rating scale (CSRS). Patients rated the intensity of cocaine high, good and bad drug effect, their desire to use cocaine and likelihood to use cocaine if it were available (desire and likelihood to use were averaged to obtain a cocaine craving score), and cocaine withdrawal on a 1 to 100 visual analog scale. Subjects also estimated the dollar value of the dose and the highest rating per session was selected as the subject's value rating.

Mood analog scale. Patients rated 16 adjectives, describing their current feelings, on a 1 to 100 visual analog scale, where above the appropriate numbers were the adjectives, not at all, mildly, moderately, and extremely. Patients were asked to estimate their feelings based on adjectives including 'high', 'anxious', 'nervous', 'irritable', 'angry', 'depressed', 'energetic', 'tired', 'drowsy', 'happy', 'sad', 'fearful', 'mellow', 'calm', 'talkative', and 'hungry'.

Plasma sampling. Blood samples, collected five times during each dose session, were spun for plasma extraction at $4{ }^{\circ} \mathrm{C}$ and immediately frozen on dry ice before transferring to storage at $-70^{\circ} \mathrm{C}$. Samples were analyzed for homovanillic acid (HVA) by HPLC and for cortisol by radioimmunoassy at the Analytical Psychopharmacology Laboratory of Mr Thomas Cooper, Nathan Kline Institute, Orangeburg, NY.

Psychophysiological measures. Blood pressure and pulse were recorded using an automated vital signs monitoring unit (ProPaq Emcore, Protocol System Inc., Beaverton, OR) with 2.5 -min and 5-min measurement interval settings.

EEG acquisition. EEG data were collected from the 19 monopolar electrode (silver chloride disc electrodes) sites of the International 10/20 System referenced to linked earlobes. In addition, transorbital electrodes were used to detect eye movement (EOG) and a neck electrode was used to monitor electrocardiogram activity (ECG). The electrophysiological data acquisition system used is a CadwellEasy EEG System 1.7 (Kennewick, WA) set with a 0.1-10 $\mu \mathrm{v}$ sensitivity, a bandpass of $0.016-70 \mathrm{~Hz}$, with a $60 \mathrm{~Hz}$ notch filter. The EEG, EOG, and EMG were recorded and digitized at $2400 \mathrm{~Hz}$ with 16-bit resolution.

EEG quantification. Using neurometric analysis, the quantitative features of the EEG were extracted, log transformed to obtain normal (Gaussian) distributions, age-regressed and $Z$-transformed relative to age-appropriate population norms recorded resting, eyes closed. Over the last two decades, neurometric qEEG norms with high testretest reliability have been derived from 550 normally functioning subjects aged 6-90 at the co-investigator's laboratory (see Prichep et al, 1996a,b; Reid et al, 2003). During recordings, raw data were continuously monitored for movement artifact, EOG activity, incidence of sleep onset, and other sources of artifact for later deletion. Captured EEG data were then recorded onto an optical disk for subsequent visual review and the selection of artifactfree epochs by a blinded rater. Artifact removal was performed by visual analysis of the raw EEG, EOG, and ECG data augmented by a computerized artifact detection algorithm. Any epoch in which any region exceeded the artifact levels was excluded from analyses. Between 1 and $2 \mathrm{~min}$ of baseline and post-dose, artifact-free EEG data, 
from pre-defined intervals, were obtained and subjected to quantitative analyses using Fast Fourier Transform (FFT). The post-dosing recording periods were: minute $0-5$ (eyes open), minute 5-10 (eyes closed), minute 15-20 (eyes open), minute 20-25 (eyes closed), minute 35-40 (eyes open), and minute 40-45 (eyes closed). For each of the 19 monopolar derivations, the absolute and relative power were computed for the delta $(1.5-3.5 \mathrm{~Hz})$, theta $(3.5-7.5)$, alpha $(7.5-12.5 \mathrm{~Hz})$, and beta $(12.5-25 \mathrm{~Hz})$ frequency bands. Inter-hemispheric coherence and symmetry between homologous leads in the four frequency bands were also computed. The qEEG data were scaled in the metric of probability as standard deviation units ( $Z$-score) relative to the age appropriate normal population, and displayed in color-coded $Z$-score images for each condition. In estimating the confidence level of $Z$ scale values for group data, the square root of sample size and the standard deviation of each group must be taken into account. For the current study $(n=13)$, the range of $Z$-scores presented $( \pm 1.2)$ is equivalent to \pm 4.5 for an individual; thus, the significance level $p<0.05$ for this group data applies to a $Z$-score difference between active and placebo of approximately 0.5 .

\section{Statistical Analyses}

Primary outcome measures were the CSRS scores for cocaine high and cocaine craving, and the neurometric qEEG activity profiles from leads over prefrontal cortex (FP1, FP2). Secondary outcome measures included the visual analog scale items 'good drug effect', 'bad drug effect', 'anxious', 'irritable', 'energetic', and 'nervous', the estimated value (in dollars) of each dose, plasma cocaine, cortisol, and HVA levels, heart rate, and blood pressure.

The subjective and neuroendocrine effects of selfadministered cocaine were compared between active $(50 \mathrm{mg})$ and placebo $(0 \mathrm{mg})$ conditions using a $6^{*}$ (baseline, $5,10,20,40,60 \mathrm{~min}$ ) by 2 (active, placebo) repeatedmeasures ANOVA ( ${ }^{*}$ cortisol and HVA were a $5 \times 2$ model). The blood pressure and heart rate were compared using a 16 (measurement intervals) by 2 (active, placebo) repeatedmeasures ANOVA.

For the qEEG data, the $Z$-scores for each condition (cocaine, placebo) relative to the age-matched normal population were used for statistical analyses. $Z$-scores (absolute and relative power at each bandwidth) obtained from the leads over the prefrontal cortex (FP1 and FP2) during the 0-5-, 5-10-, 15-20-, and 20-25-min intervals after dosing were selected a priori for analysis using Hotellings $T^{2}$ statistics to compare the multivariate differences during the period of self-reported cocaine high. In addition, all $Z$-score maps (absolute and relative power, coherence, and asymmetry) acquired during the 0-5-, 5-10-, 15-20- and 20-25-min intervals after dosing were visually inspected and areas of interest were selected based on: (a) distinct differences $(\Delta Z \geqslant 0.4)$ between dose conditions, and (b) previous findings of activation in the same region from earlier brain imaging studies (Breiter et al, 1997; Christensen et al, 2000; Bartzokis et al, 1999). Brain regions matching the criteria for both (a) and (b) were analyzed using Hotellings $T^{2}$ statistics, resulting in a conservative analytical model with a reduced number of tests computed.
Regional groupings of leads (eg FP1, FP2 = prefrontal cortex; FP1, FP2, F3, F4, F7, F8, Fz = frontal cortex; O1, O2, T5, T6=occipital/posterior temporal cortex) were consistent across all qEEG profiles compared. Comparisons with baseline were always made in the same recording condition: eyes open $v s$ min $0-5$ or min 15-20; eyes closed $v s$ min $5-10$ or min $20-25$.

The peak increase (relative to baseline) in the subjective and physiological measures which were significantly different between cocaine and placebo were calculated for correlation analyses: cocaine high, cocaine craving, ratings of 'nervous' and 'good drug effect', heart rate, plasma cortisol, and plasma cocaine levels. The peak increase in these subjective ratings and physiological measures, the estimated value of dose, and plasma cocaine levels were then compared using Pearson's correlation analyses. These peak increase measures were also compared with qEEG data $Z$-scores using Canonical (qEEG absolute and relative power) and Pearson's (qEEG coherence) correlations. As the peak increase in the subjective and physiological response measures were within the first $10 \mathrm{~min}$ in nearly all cases (with one exception for plasma cortisol), $Z$ transformed qEEG data from the 0-5- and 5-10-min postdosing time intervals were used for the correlation analyses. To further limit the number of tests computed, the selection of qEEG data was limited to absolute and relative power and coherence at FP1 and FP2.

\section{RESULTS}

\section{Subjective Responding}

All subjects self-administering $50 \mathrm{mg}$ cocaine base reported an increase in cocaine high, with the effects lasting for approximately $20 \mathrm{~min}$ in most individuals. Peak plasma levels were obtained at $10 \mathrm{~min}$ post-dose (plasma level: cocaine $=104 \pm 22 \mathrm{ng} / \mathrm{ml}$, used stem placebo: $9.58 \pm 5.83 \mathrm{ng} /$ $\mathrm{ml})$. Analysis of the full time course of the test procedures showed a significant effect of cocaine dose on self-reported cocaine high $(\mathrm{F}(5,60)=33.477, p<0.001)$ (Figure 1a). There was also a significant increase in cocaine craving $(\mathrm{F}(5,60)=2.298, p<0.05) \quad$ (Figure $1 \mathrm{~b})$, and subjective ratings for 'good drug effect' $(\mathrm{F}(5,60)=30.252, p<0.001)$ (Figure 1c), 'nervous' $(\mathrm{F}(5,60)=4.037, p<0.01)$ (Figure 1d), and 'high' $(\mathrm{F}(5,60)=30.252, p<0.001)$ (data not shown) following cocaine. Cocaine high was positively correlated with plasma cocaine levels $(r=0.60, p<0.05)$, 'good drug effect' $(r=0.95, p<0.01)$, and the estimated value of the active dose $(11.3 \pm 3.2$ dollars $)(r=0.56, p<0.05)$. 'Good drug effect' was correlated with the estimated value of the active dose $(r=0.71, p<0.01)$ and (at a trend level) with plasma cocaine levels $(r=0.58, p=0.060)$.

There were no effects of placebo dosing on self-reported cocaine high or cocaine craving, or any of the other subjective effects elicited by cocaine. In contrast, a significant increase in subjective ratings for 'irritable' $(\mathrm{F}(5,60)=2.723, p<0.05)$ was noted (data not shown). Though a minor increase in 'high' immediately following placebo was indicated in some patients, within-group comparison of baseline and minute 5 post-dose ratings was not significant $(t=4.462, p=0.102)$. Most patients 
(10/13) determined they had received placebo within the first $5 \mathrm{~min}$, confirmed by a one-item post-dose question at the 5 -min post-dose time point.
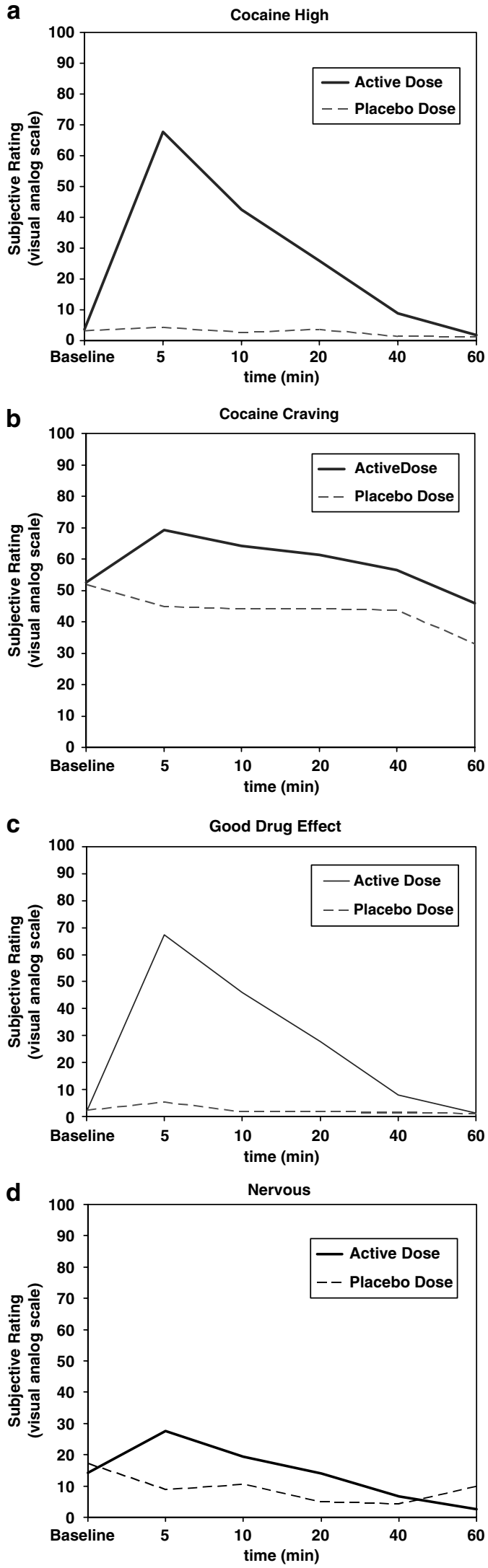

\section{Physiological Effects}

Smoked cocaine base produced a significant increase in heart rate $(\mathrm{F}(18,198)=6.759, p<0.001)$, reaching a peak of 101 beats per minute $(\mathrm{bpm})$ at minute 5 post-dose and returning to baseline within $20 \mathrm{~min}$ in most individuals (Figure 2). Systolic and diastolic blood pressure showed a mild increase following cocaine (data not shown), though neither effect was statistically significant given the briefness of the effect (systolic-F $(18,198)=1.088, p=0.362$; diastolic $-\mathrm{F}(18,198)=1.291, \quad p=0.189)$. Plasma cortisol levels displayed a sustained increase, but HVA levels were unchanged, following cocaine (cortisol $-\mathrm{F}(4,44)=5.453$, $p<0.001 ; \quad$ HVA $-\mathrm{F}(4,32)=0.094, p=0.984) \quad($ Table 1$)$.

Table I Plasma Levels of Cortisol and HVA Following Smoked Cocaine Base or Placebo

\begin{tabular}{lccccc}
\hline & Baseline & Min 10 & Min 20 & Min 40 & Min 60 \\
\hline $\begin{array}{l}\text { Cortisol }(\mu g / d) \\
\text { Cocaine }(50 \mathrm{mg})\end{array}$ & $11.0 \pm 3.6$ & $13.6 \pm 1.5$ & $15.3 \pm 5.8$ & $14.2 \pm 6.2$ & $10.8 \pm 3.2$ \\
Placebo & $11.4 \pm 4.6$ & $10.9 \pm 3.7$ & $10.4 \pm 3.7$ & $9.1 \pm 3.6$ & $9.1 \pm 4.3$ \\
& & & & & \\
HVA (ng/ml) & & & & & \\
$\begin{array}{l}\text { Cocaine }(50 \mathrm{mg}) \\
\text { Placebo }\end{array}$ & $12.2 \pm 5.2$ & $12.7 \pm 4.4$ & $12.1 \pm 4.9$ & $12.3 \pm 4.4$ & $12.3 \pm 4.0$ \\
\hline
\end{tabular}

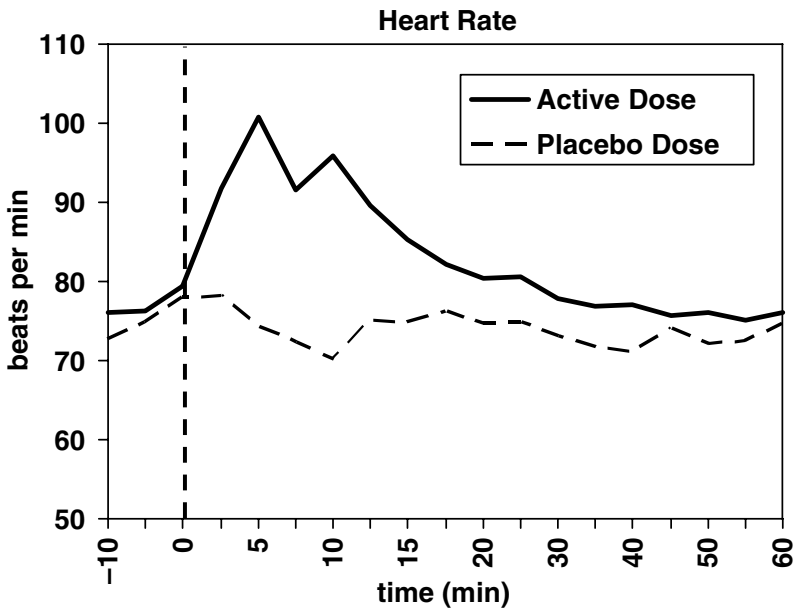

Figure 2 The effects of a smoked cocaine base $(50 \mathrm{mg}$ crack cocaine in a used stem) vs placebo ( $0 \mathrm{mg}$ crack cocaine in a used stem) on heart rate in cocaine-dependent patients $(N=13)$. Heart rate was monitored with chest electrodes via an automated device (Propack) according to the following schedule: 5-min intervals during baseline, 2.5-min intervals minutes 0-20 following dosing, and 5-min intervals minutes 20-60 following dosing. All effects were compared with placebo $(p<0.0 \mathrm{I})$ using repeated-measures, between-group, ANOVA comparisons.

Figure I The subjective effects of a smoked cocaine base $(50 \mathrm{mg}$ crack cocaine in a used stem) vs placebo (0 mg crack cocaine in a used stem) in cocaine-dependent patients $(N=13)$. Subjective ratings, measured by visual analog scale, were collected 10 min prior to dosing (baseline) and at time points represented thereafter for I $\mathrm{h}$ following dosing. In (a) the effects on cocaine high $(p<0.0 \mathrm{l})$, in (b) the effects on cocaine craving $(p<0.05)$, in (c) the effects on good drug effect $(p<0.0 \mathrm{I})$, and in $(d)$ the effects on nervous $(p<0.01)$ are represented. All effects were significantly different from active placebo based on repeated-measures, betweengroup, ANOVA comparisons. 
There were no effects of placebo dosing on heart rate, blood pressure, or plasma levels of cortisol and HVA.

\section{qEEG Measures}

Based upon visual analysis of the $Z$-score maps, smoked cocaine base $(50 \mathrm{mg})$ produced an apparent increase in absolute EEG power recorded from scalp electrodes over the prefrontal cortex (FP1 and FP2). This effect was clearest in the eyes closed recording condition, reaching a maximum during the minute 5-10 post-dose recording period (Figure 3a) and then subsiding gradually across the minute 15-25 (Figure 3b) and minute 35-45 post-dose recording periods. Hotellings $T^{2}$ test comparisons of absolute power over FP1 and FP2 are displayed in Table 2. Comparison of cocaine dose $v s$ baseline revealed a

a

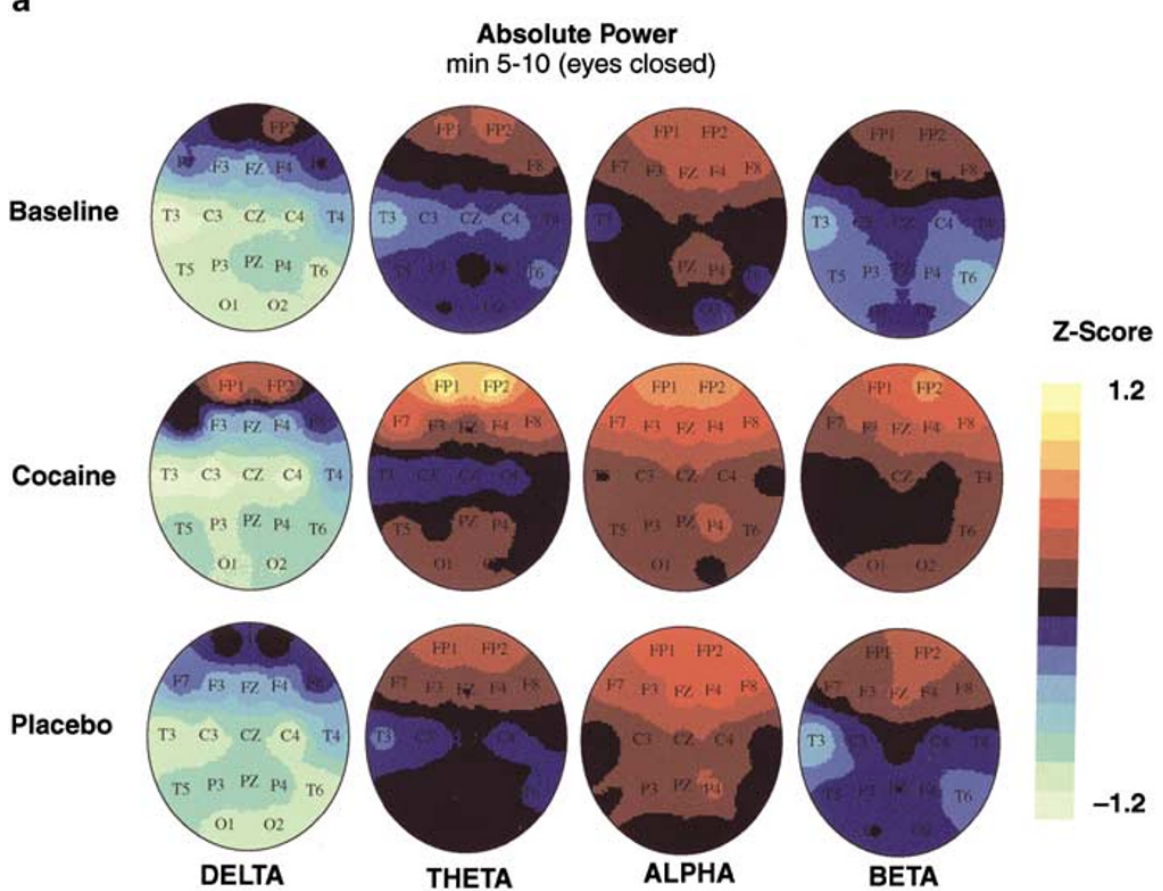

b

Absolute Power $\min 20-25$ (eyes closed)
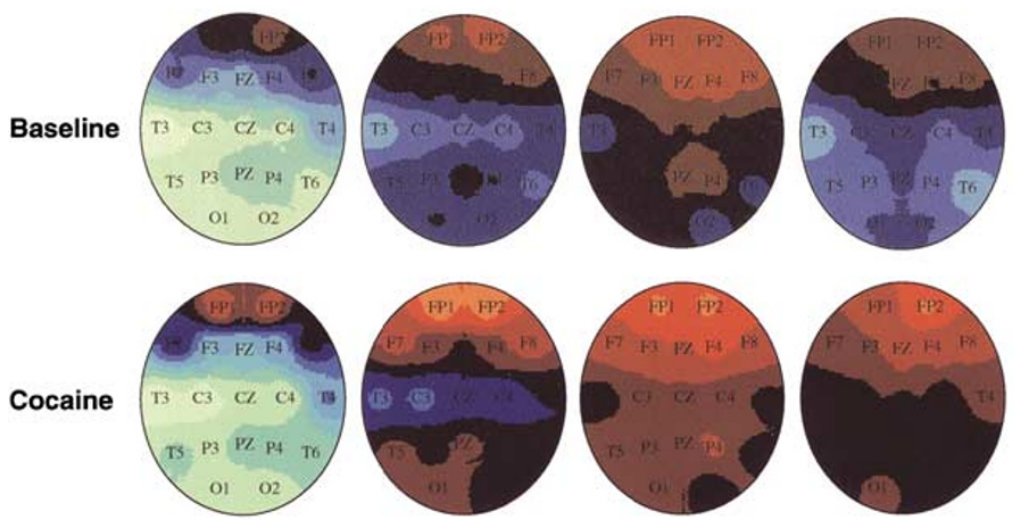

Z-Score
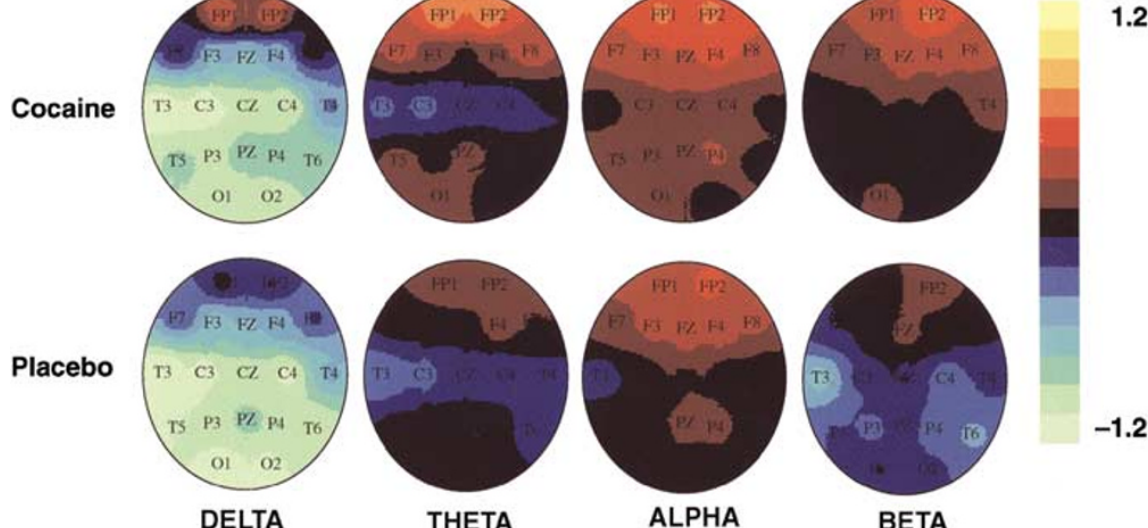

Figure 3 Group average topographic head maps of Z-scores for absolute power in delta $(1.5-3.5 \mathrm{~Hz})$, theta (3.5-7.5), alpha (7.5-12.5 Hz), and beta $(12.5-25 \mathrm{~Hz})$ frequency bands averaged across the 5-min baseline and the (a) minute 5-10 and (b) minute 20-25 EEG recording periods following smoked cocaine $(50 \mathrm{mg})$ or placebo. All recordings were collected in the eyes closed condition. Color coding, in steps corresponding to those shown in the Z-scale (range \pm 1.2 ), is proportional to the difference in Z-scores from a healthy normative population. 
significant increase in power at minute 5-10 (theta and beta), minute 15-20 (alpha), and minute 20-25 (theta, alpha, beta) following dosing. Theta power also displayed trend level increase $(p=0.063)$ during minute $15-20$ following dosing. Correlation analyses comparing cocaineinduced subjective and physiological responding with absolute power over FP1 and FP2 at minute 0-5 (eyes open) revealed a positive correlation between delta power and cocaine craving, between alpha power and 'nervous', and between theta power and plasma cortisol (Table 3a). Similar correlation analyses at minute 5-10 (eyes closed) revealed a positive correlation between theta power and 'good drug effect', and between alpha power and 'nervous'. There was also a trend level positive correlation of delta $(p=0.070)$ and theta power $(p=0.070)$ with plasma cocaine levels (Table 3b).
Based upon visual analysis of the $Z$-score maps, smoked cocaine base $(50 \mathrm{mg})$ produced an apparent increase in relative delta and theta EEG power recorded from scalp electrodes over the prefrontal cortex (FP1 and FP2). This effect was brief, seen only during the minute $0-5$ (eyes open) and minute 5-10 (eyes closed) post-dose recording periods (data not shown). Hotellings $T^{2}$ test comparisons of relative power over FP1 and FP2 are displayed in Table 2. Comparison of cocaine dose $v s$ baseline did not reveal a significant increase in relative delta and theta power, though an increase in relative beta power during the minute 20-25 post-dose recording period was found. Correlation analyses comparing cocaine-induced subjective and physiological responding with relative power over FP1 and FP2 at minute 5-10 (eyes closed) revealed a positive correlation between relative theta power and plasma cocaine levels (Table $3 \mathrm{~b}$ ).

Table 2 Cocaine-Induced qEEG Power and Coherence at FPI and FP2: Hotellings T2 Tests

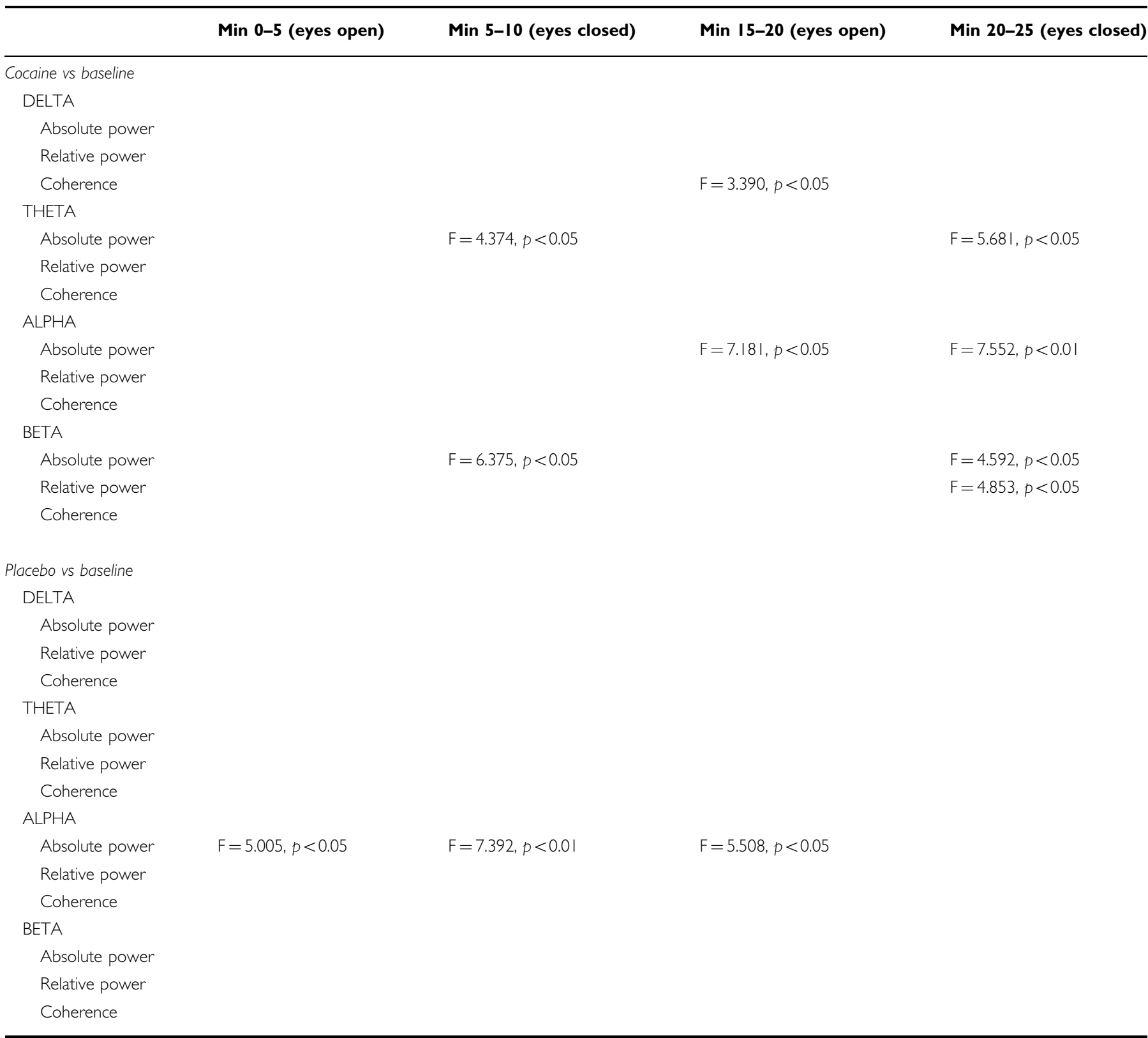


Table 3 Cocaine-Induced qEEG (FPI and FP2), Subjective, and Physiological Responding: Correlation Analyses

\begin{tabular}{|c|c|c|c|c|c|c|c|}
\hline & Cocaine high & Good drug & Craving & Nervous & Cortisol & Heart rate & Plasma cocaine \\
\hline \multicolumn{8}{|l|}{ (a) Min 0-5 (eyes open) } \\
\hline \multicolumn{8}{|l|}{ DELTA } \\
\hline \multirow[t]{2}{*}{ Absolute power } & & & $r=0.76$ & & & & \\
\hline & & & $p<0.05$ & & & & \\
\hline Coherence & & & & & $r=0.69$ & & \\
\hline \multirow[t]{2}{*}{ Absolute power } & & & & & $r=0.82$ & & \\
\hline & & & & & $p<0.05$ & & \\
\hline \multicolumn{8}{|l|}{ Relative power } \\
\hline Coherence & & & & & $r=0.85$ & & \\
\hline \multicolumn{8}{|l|}{ Relative power } \\
\hline \multirow[t]{2}{*}{ Coherence } & & & & & $r=0.77$ & & \\
\hline & & & & & $p<0.05$ & & \\
\hline \multicolumn{8}{|l|}{ BETA } \\
\hline \multicolumn{8}{|l|}{ Absolute power } \\
\hline \multicolumn{8}{|l|}{ Relative power } \\
\hline \multicolumn{8}{|l|}{ Coherence } \\
\hline \multicolumn{8}{|l|}{ (b) Min 5-10 (eyes closed) } \\
\hline \multicolumn{8}{|l|}{ DELTA } \\
\hline & & $p<0.05$ & & & & & \\
\hline Relative power & & & & & & & $r=0.72$ \\
\hline & & & & & & & $p<0.05$ \\
\hline Coherence & & & & $r=-0.77$ & & & \\
\hline & & & & $p<0.05$ & & & \\
\hline ALPHA & & & & & & & \\
\hline Absolute power & & & & $r=0.71$ & & & \\
\hline & & & & $p<0.05$ & & & \\
\hline Relative power & & & & & & & \\
\hline Coherence & & & & & & & \\
\hline BETA & & & & & & & \\
\hline Absolute power & & & & & & & \\
\hline Relative power & & & & & & & \\
\hline Coherence & & & & & & $r=-0.60$ & \\
\hline & & & & & & $p<0.05$ & \\
\hline
\end{tabular}

Based on visual analysis of the $Z$-score maps, smoked cocaine base $(50 \mathrm{mg}$ ) produced an apparent increase in EEG coherence recorded from scalp electrodes over the pre- frontal cortex (FP1 and FP2). This effect was clearest in the eyes closed recording condition in the theta and delta bandwidths, reaching a maximum during the minute 5-10 


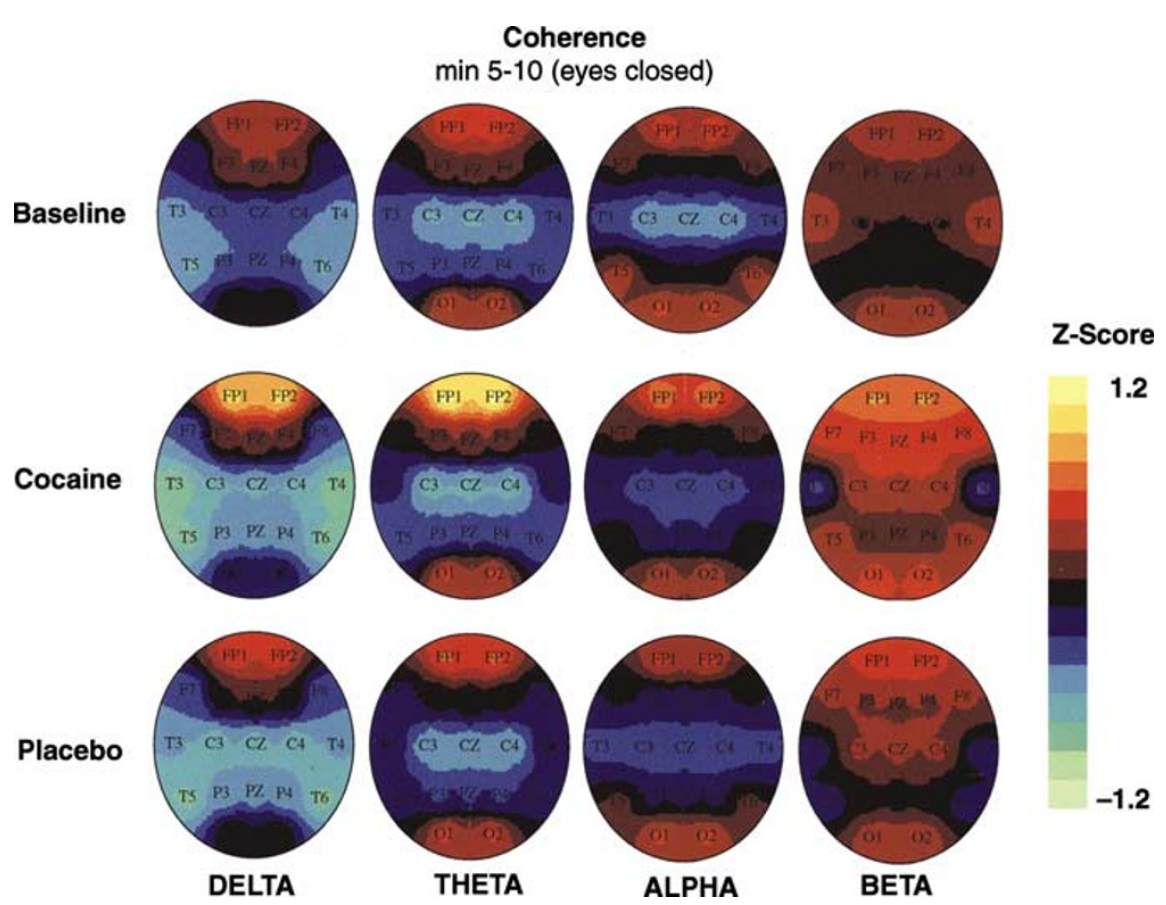

Figure 4 Group average topographic head maps of Z-scores for EEG coherence in delta $(1.5-3.5 \mathrm{~Hz})$, theta $(3.5-7.5)$, alpha $(7.5-12.5 \mathrm{~Hz})$, and beta $(12.5-25 \mathrm{~Hz})$ frequency bands averaged across the 5-min baseline and the minute 5- 10 EEG recording period following smoked cocaine (50 mg) or placebo. All recordings were collected in the eyes closed condition. Color coding, in steps corresponding to those shown in the Z-scale (range \pm 1.2 ), is proportional to the difference in Z-scores from a healthy normative population.

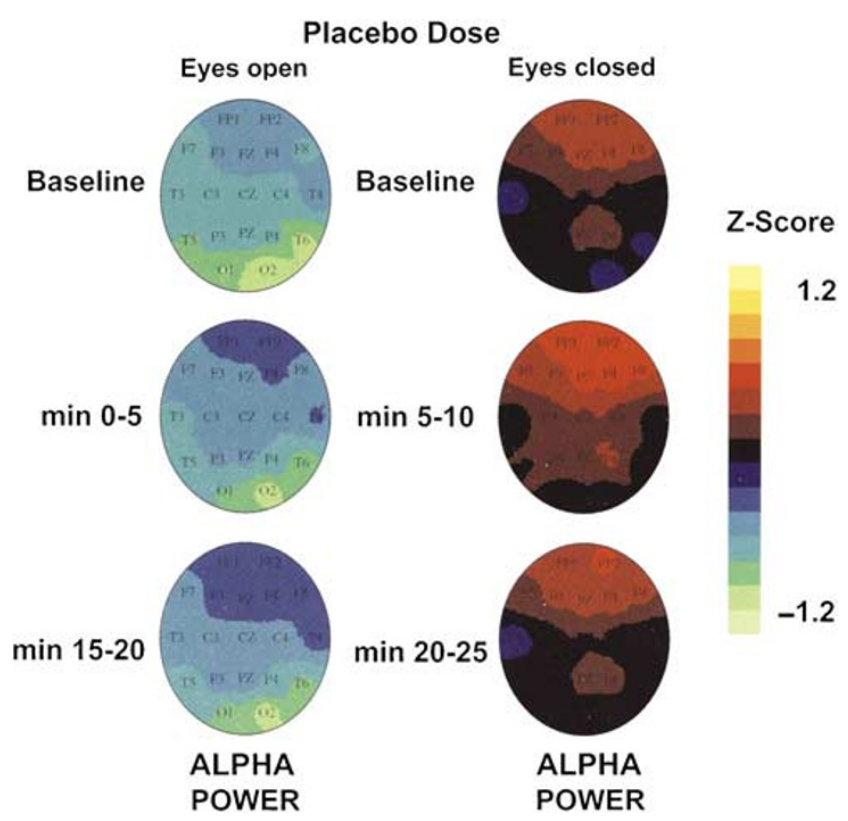

Figure 5 Group average topographic head maps of Z-scores for absolute power in the alpha $(7.5-12.5 \mathrm{~Hz})$ frequency band averaged across the baseline and the minute $0-5$, minute $5-10$, minute $15-20$, and minute 20-25 EEG recording periods following smoked placebo. Recordings were collected in the eyes open or eyes closed conditions, as indicated. Color coding, in steps corresponding to those shown in the Z-scale (range \pm I.2), is proportional to the difference in $Z$-scores from a healthy normative population.

post-dose recording period (Figure 4) and then subsiding gradually across the minute $15-25$ and minute $35-45$ postdose recording periods. Hotellings $T^{2}$ test comparisons of coherence over FP1 and FP2 are displayed in Table 2. Comparison of cocaine dose $v s$ baseline revealed a significant increase in delta coherence during minute 15-20 following dosing. Correlation analyses comparing cocaine-induced subjective and physiological responding with coherence over FP1 and FP2 at minute $0-5$ (eyes open) revealed a positive correlation of delta, theta, and alpha coherence with plasma cortisol (Table 3a). Similar correlation analyses at minute 5-10 (eyes closed) revealed a negative correlation of delta and theta coherence with 'nervous', and a negative correlation between beta coherence and heart rate (Table $3 b$ ).

Based upon visual analysis, placebo dosing had a minor effect on qEEG, indicating a persistent increase in alpha power recorded from scalp electrodes over the prefrontal cortex (FP1, FP2) (Figure 5). Hotellings $T^{2}$ test comparisons of absolute and relative power, and coherence, over FP1 and FP2, placebo $v s$ baseline, are displayed in Table 2. A significant increase in alpha power at minute $0-5$, minute $5-10$, and minute 15-20 following placebo dosing was noted. There was also a trend effect increase in alpha power $(p=0.056)$ at minute $20-25$. Comparisons of coherence and relative power over FP1 and FP2 revealed no significant effects of placebo dosing.

\section{DISCUSSION}

The present findings demonstrate that self-administration of cocaine base (crack cocaine) produces alterations in neurophysiological activity in cocaine-dependent patients, as evidenced by enhanced GEEG power and coherence over the frontal pole scalp electrodes. Moreover, these changes in 
qEEG profiles followed a time course similar to the subjective effects of cocaine. These data confirm previous studies on the acute effects of cocaine on EEG in humans that have reported an increase in delta, alpha, and beta power following cocaine (Berger, 1931; Herning et al, 1985; Lukas and Mendelson, 1988) and extend them by neuroanatomically mapping the effects of cocaine to the frontal poles, over the prefrontal cortex. The localization of the acute effects of cocaine over the prefrontal cortex is consistent with previous MRI and PET studies on cocaine effects in humans (Breiter et al, 1997, Bartzokis et al, 1999) and primates (Howell et al, 2002; Porrino et al, 2002), showing alterations of neuronal activity, metabolism, and blood flow in the limbic system.

Subjects in the current study received a medium dose of cocaine, reaching approximately $100 \mathrm{ng} / \mathrm{ml}$ cocaine plasma levels within the first $10 \mathrm{~min}$, similar to that following smoked cocaine $(50 \mathrm{mg}$ ) in a binge protocol (Ward et al, 1997), but less than that following smoked cocaine in protocols employing corncob pipe $(50 \mathrm{mg}$ ) (Foltin and Fischman, 1991) or thermal nebulizer (35-40 mg) (Hatsukami et al, 1990, 1994) devices. In addition, the levels obtained in the current study were generally lower than reported in previous neuroimaging studies which employed i.v. cocaine (30-40 mg) (Breiter et al, 1997; Kaufman et al, 1998a; Christensen et al, 2000; see Mendelson et al, 1999), though the prior EEG studies on acute cocaine effects did not report the plasma cocaine levels (Herning et al, 1985, 1994; Lukas et al, 1990; Lukas, 1991). In general, the levels of cocaine obtained through the smoking procedures in the current study were moderately variable, which likely reflects the nature of a self-administered, smoked dose of cocaine base.

In the present study, there were clear effects of cocaine smoking on ratings of cocaine high and 'good drug effect', which were rapid and somewhat short lasting. These effects were accompanied by increased ratings of 'nervous' and cocaine craving, which were more moderate but persisted at or near their peak levels for a longer duration. These findings are in agreement with prior cocaine smoking studies (Foltin and Haney, 2000; Ward et al, 1997; Hart et al, 2004), and extend them by demonstrating the time course of subjective effects over a period of $60 \mathrm{~min}$ after dosing. Moreover, evidence for a more prolonged effect on cocaine craving and nervousness is consistent with previous neuroimaging study that reported a short high followed by a longer period of cocaine craving following i.v. dosing (Breiter et al, 1997). Analysis of the placebo (CS +) group did not provide any evidence for a conditioned stimulus effect. On the contrary, the higher ratings for 'irritable' suggest that subjects did not experience any form of psychosomatic cocaine high. Interestingly, patients in the placebo condition did not report an increase in cocaine craving despite not receiving a dose of cocaine when they prepared for, and were instructed to expect, one. This suggests that cocaine use may lead to craving and continued drug use (binge use) based on a pharmacologically, as opposed to contextually, mediated priming effect.

The cardiovascular measures collected in this study demonstrated a clear effect of cocaine smoking on heart rate. In contrast, the effects of cocaine on blood pressure were small in the current study and did not replicate the robust effects of cocaine reported in previous studies on smoked cocaine (Foltin and Haney, 2000; Foltin and Fischman, 1991; Hatsukami et al, 1990) or i.v. cocaine (Breiter et al, 1997; Christensen et al, 2000). The effects of cocaine on heart rate were similar to that reported following i.v. cocaine in previous neuroimaging studies (Breiter et al, 1997; Kaufman et al, 1998a; Christensen et al, 2000). This increase in heart rate, approximately $25 \mathrm{bpm}$, was somewhat greater than that obtained in a smoked cocaine study that employed the same dose $(50 \mathrm{mg})$ and smoking instruments, paired with conditioned cues $(\mathrm{CS}+$ ) (Foltin and Haney, 2000). However, in this earlier study, conditioned cues followed by placebo produced a minor but significant increase in the heart rate (approximately $7 \mathrm{bpm}$ ) (Foltin and Haney, 2000), while in the present study cocaine cues and dosing expectations $(\mathrm{CS}+)$ followed by placebo had no effect on heart rate.

Plasma cortisol levels demonstrated a robust increase following cocaine. While it is well known that cocaine stimulates cortisol secretion (Hopper et al, 2004; Ward et al, 1999), evidence for the involvement of cortisol and the hypothalamic-pituitary-adrenal (HPA) axis in cocaine craving and reward has been somewhat contradictory (Ward et al, 1999; Lee et al, 2003; Sinha et al, 2003; Elman et al, 2003; Broadbear et al, 2004). Indeed, cocaine-induced plasma cortisol levels did not correlate with any of the subjective ratings employed in this study. The increase in cortisol was, however, positively correlated with cocaineinduced delta, theta, and alpha EEG coherence recorded from the frontal pole electrodes. Previous studies have reported that cortisol administration promotes an increase in delta and theta activity (Friess et al, 2004), though investigations on endogenous levels have demonstrated delta power and coherence to be inversely related to cortisol levels (Brandenberger, 2003; Schutter et al, 2004). Our findings extend this work by indicating that EEG and the HPA axis are activated in a coordinated fashion in response to cocaine administration.

Previous studies on the effects of cocaine on human EEG have reported an increase in beta, alpha, and delta bandwidth activity (Berger, 1931; Herning et al, 1985, 1994; Lukas et al, 1990; Lukas, 1991). In the current study, evidence for an increase in beta and alpha power, relative beta power, and delta coherence are consistent with these reports. The finding of an increase in theta power further expands upon these prior studies. In the previous studies, the functional relevance of these changes in EEG was attributed to higher frequency bandwidth EEG. For example, enhanced alpha power was positively correlated with heart rate and systolic blood pressure (Herning et al, 1994), and enhanced beta power was positively correlated with heart rate and cocaine plasma levels (Herning et al, $1985,1994)$. Studies by Lukas et al $(1988,1990,1995)$ have consistently reported an increase in alpha activity after cocaine, as well as other drugs of abuse, concurrent with episodic periods of euphoria. Similar to the clinical data, some animal studies have reported that acute cocaine and amphetamine treatment results in enhanced alpha activity, which was strongest in the alpha-1 bandwidth $(7-9.5 \mathrm{~Hz})$ and was blocked by D1 dopamine antagonists (Stahl et al, 1997; Ferger et al, 1994). 
In the present study, cocaine-induced alpha power was correlated with ratings of 'nervous', and alpha coherence was correlated with cortisol levels. This suggests that alpha EEG activity is associated with the autonomic arousal and anxiety-producing effects of cocaine. Interestingly, analysis of the placebo $(\mathrm{CS}+)$ condition revealed an increase in alpha power for the first $20-25 \mathrm{~min}$ after dosing when compared with baseline recordings. It might be expected that this increase in alpha power following placebo represents a conditioned drug-like effect; however, this occurred in subjects reporting an increase in 'irritable' levels and no cocaine high following dosing. Thus, findings in the present study do not support the suggestion that alpha power is associated with the rewarding effects of cocaine (Herning et al, 1994; Lukas et al, 1990; Lukas, 1991). The studies by Lukas and colleagues differed from the current study in their measurement of drug-induced euphoria, in which episodic moments of drug reward were determined by a joystick device as opposed to discrete cross sections of time after dose. In fact, when averaged across the first $20 \mathrm{~min}$ following dosing, marijuana produced an overall decrease in alpha power (Lukas et al, 1995) and a high dose of cocaine had only weak effects on alpha (Lukas et al, 1990). In the study by Herning et al (1994), EEG measures were averaged across a lengthy 30 -min post-dose period, which could obscure the immediate rewarding effects of cocaine on EEG and reward. Indeed, it is well established that cocaine-induced euphoria is rapid and relatively short lasting (Foltin et al, 1990; Foltin and Fischman, 1991; Foltin and Haney, 2000).

The qEEG effects of cocaine in the present study, based on neurometric derived $Z$-scores, were most pronounced in the lower-frequency EEG bandwidths delta and theta. These effects were strongest in the first $10 \mathrm{~min}$ following dosing, evident in absolute power and coherence and were focused over the frontal pole electrodes. These findings are supported by two previous clinical studies reporting cocaine-induced delta power (Herning et al, 1985) and morphine-induced theta power (Phillips et al, 1994). In animal studies, one group has reported cocaine-induced hippocampal theta activity, which was blocked by both D1 dopamine and cholinergic receptor antagonists (Yabase et al, 1990; Yoshida et al, 1993). The involvement of dopamine in this effect of cocaine on EEG is consistent with the present finding of qEEG alterations over a dopamineinnervated region of the cortex, the prefrontal cortex. The increase in slow wave coherence likely represents increased synchronization of neuronal firing, possibly mediated by the ascending cholinergic subcortical-cortical projections from the thalamus that are critical to the generation of slow wave EEG rhythm (see Steriade et al, 1990, 1993).

Low-frequency EEG activity profiles recorded from the frontal poles were correlated with a number of subjective responses measures, as well as plasma cocaine levels, during the first $10 \mathrm{~min}$ following dosing. Theta power was positively correlated with ratings of 'good drug effect', while theta and delta coherence were negatively correlated with ratings of 'nervous'. Plasma cocaine levels were positively correlated with relative theta power and (at a trend level) with absolute delta and theta power. This pattern of association between subjective drug effects, plasma cocaine levels, and EEG indicates that delta and theta EEG activity may reflect the rewarding properties of cocaine. Interestingly, delta power during the first $5 \mathrm{~min}$ following cocaine was also correlated with increased ratings of cocaine craving. This finding is consistent with our previous studies on cue-induced cocaine craving which reported an increase in delta power over the prefrontal cortex during the guided imagery phase of cocaine cue presentation (Reid et al, 2003). Others have reported a decrease in alpha power during cocaine cue exposure (Liu et $a l, 1998)$ and a negative correlation between cocaineinduced alpha power and the subjective response item 'use again' (Herning et al, 1994). These data are consistent with the suggestion that lower-frequency EEG activity is associated with cocaine craving. It should be noted, however, that a cocaine-like high is also reported during cocaine cue exposure (Reid et al, 2003, 2004). Further studies comparing cocaine cue- and drug-elicited subjective responding and AEEG will be needed to determine the relative contribution of delta and theta EEG to cocaine reward and craving.

Prior neuroimaging studies have provided extensive evidence that acute cocaine produces reductions in cerebral blood volume (Kaufman et al, 1998a,b), cerebral blood flow (Wallace et al, 1996; Gollub et al, 1998), and glucose metabolism (Bartzokis et al, 1999). While the neuranatomical specificity of these effects are yet to be characterized in humans, PET and autoradiography studies in primates have both demonstrated similar cocaine effects in the prefrontal cortex (Porrino et al, 2002; Howell et al, 2002), consistent with the location of the qEEG effects observed in the present study. Prior studies comparing qEEG with cerebral blood flow have demonstrated a negative correlation between cortical cerebral blood flow and both delta (Tolonen and Sulg, 1981; Rodriguez et al, 1999) and theta (Mattia et al, 2003) power. In both the acute cocaine and qEEG studies, the measurements of cerebral blood flow were based on similar flow-sensitive inversion recovery functional MRI (Gollub et al, 1998; Mattia et al, 2003) and 99m Tc-HMPAO SPECT (Wallace et al, 1996; Rodriguez et al, 1999) recording techniques. Based on these results obtained using comparable methodologies, it can be predicted that acute cocaine would produce a decrease in cerebral blood flow accompanied by an increase in delta and theta EEG activity in the prefrontal cortex. Indeed, the findings on cocaine-induced qEEG in the present study are consistent with this suggestion.

\section{ACKNOWLEDGEMENTS}

This study was supported by NIDA R21 DA 12277 to Malcolm S Reid, NIDA RO1 DA07707 to Leslie Prichep, and by grant NIH/NCRR MO1RR00096 to the New York University School of Medicine, General Clinical Research Center.

\section{REFERENCES}

Alper KR, Chabor RJ, Kim AH, Prichep LS, John ER (1990). Quantitative EEG correlates of crack cocaine dependence. Psychiatr Res Neuroimag 35: 95-105.

Bartzokis G, Beckson M, Newton T, Mandelkern M, Mintz J, Foster JA et al (1999). Selegiline effects on cocaine-induced changes in 
medial temporal lobe metabolism and subjective ratings of euphoria. Neuropsychopharmacology 20: 582-590.

Berger H (1931). Arch Psychiat Nervenkr 94: 16-30 Translated in Gloor P (ed) (1969). Hans Berger on the electroencephalogram of man. Electroencephalogr Clin Neurophysiol (Suppl 28). Elsevier: Amsterdam, pp 95-132.

Berger SP, Hall SM, Mickalian JD, Reid MS, Crawford CA, Delucchi KL et al (1996). Haloperidol antagonism of cue-elicited cocaine craving. Lancet 347: 504-508.

Brandenberger $G$ (2003). The ultradian rhythm of sleep: diverse relations with pituitary and adrenal hormones. Rev Neurol 159: 6S5-6S10.

Breiter HC, Gollub RL, Weisskoff RM, Kennedy DN, Makris N, Berke JD et al (1997). Acute effects of cocaine on human brain activity and emotion. Neuron 19: 591-611.

Broadbear JH, Winger G, Woods JH (2004). Self-administration of fentanyl, cocaine and ketamine: effects on the pituitaryadrenal axis in rhesus monkeys. Psychopharmacology 176: 398-406.

Christensen JD, Kaufman MJ, deB Frederick B, Rose SL, Moore CM, Lukas SE et al (2000). Proton magnetic resonance spectroscopy of human basal ganglia: response to cocaine administration. Biol Psychiatry 48: 685-692.

Elman I, Lukas SE, Karlsgodt KH, Gasic GP, Breiter HC (2003). Acute cortisol administration triggers craving in individuals with cocaine dependence. Psychopharmacol Bull 37: 84-89.

Ferger B, Kropf W, Kuschinsky K (1994). Studies on electroencephalogram (EEG) in rats suggest that moderate doses of cocaine or $d$-amphetamine activate $\mathrm{D}_{1}$ rather than $\mathrm{D}_{2}$ receptors. Psychopharmacology 114: 297-308.

Foltin RW, Fischman MW (1991). Smoked and intravenous cocaine in humans: acute tolerance, cardiovascular and subjective effects. J Pharmacol Exp Ther 257: 247-261.

Foltin RW, Fischman MW, Nestadt G, Stromberger H, Cornell EE, Pearlson GD (1990). Demonstration of naturalistic methods for cocaine smoking by human volunteers. Drug Alcohol Depend 26: $145-154$.

Foltin RW, Haney M (2000). Conditioned effects of environmental stimuli paired with smoked cocaine in humans. Psychopharmacology 149: 24-33.

Friess E, Tagaya H, Grethe C, Trachsel L, Holsboer F (2004). Acute cortisol administration promotes sleep intensity in man. Neuropsychopharmacology 29: 598-604.

Gollub RL, Breiter HC, Kantor H, Kennedy D, Gastfriend D, Mathew RT et al (1998). Cocaine decreases cortical cerebral blood flow but does not obscure regional activation in functional magnetic resonance imaging in human subjects. J Cerebr Blood Flow Metab 18: 724-734.

Hart CL, Ward AS, Collins ED, Haney M, Foltin RW (2004). Gabapentin maintenance decreases smoked cocaine-related subjective effects, but not self-administration by humans. Drug Alcohol Depend 73: 279-287.

Hatsukami D, Keenan R, Carroll M, Colon E, Geiske D, Wilson B et al (1990). A method for delivery of precise doses of smoked cocaine-base to humans. Pharmacol Biochem Behav 36: 1-7.

Hatsukami DK, Pentel PR, Glass J, Nelson R, Brauer LH, Crosby R et al (1994). Methodological issues in the administration of multiple doses of smoked cocaine-base in humans. Pharmacol Biochem Behav 47: 531-540.

Herning RI, Glover BJ, Koeppl B, Phillips RL, London ED (1994). Cocaine-induced increases in EEG alpha and beta activity: evidence for reduced cortical processing. Neuropsychopharmacology 11: 1-9.

Herning RI, Guo X, Better WE, Weinhold LL, Lange WR, Cadet JL et al (1997). Neurophysiological signs of cocaine dependence: increased electroencephalogram beta during withdrawal. Biol Psychiatry 41: 1087-1094.
Herning RI, Jones RT, Hooker WD, Mendelson J, Blackwell L (1985). Cocaine increases EEG beta: a replication and extension of Hans Berger's historic experiments. Electroencephalogr Clin Neurophysiol 60: 470-477.

Hopper JW, Karlsgodt KH, Adler CM, Macklin EA, Lukas SE, Elman I (2004). Effcts of acute cortisol and cocaine administration on attention, recall and recognition task performance in individuals with cocaine dependence. Hum Psychopharmacol 19: 511-516.

Howell LL, Hoffman JM, Votaw JR, Landrum AM, Wilcox KM, Lindsey KP (2002). Cocaine-induced brain activation determined by positron emission tomography neuroimaging in conscious rhesus monkeys. Psychopharmacology 159: 154-160.

Kaufman MC, Levin JM, Maas LC, Rose SL, Lukas SE, Mendelson $\mathrm{JH}$ et al (1998a). Cocaine decreases relative cerebral blood volume in humans: a dynamic susceptibility contrast magnetic resonance imaging study. Pharmacology 138: 76-81.

Kaufman MJ, Levin JM, Ross MH, Lange N, Rose SL, Kukes TJ et al (1998b). Cocaine-induced cerebral vasoconstriction detected in humans with magnetic resonance angiography. JAMA 279: $376-380$

Lee B, Tiefenbacher S, Platt DM, Spealman RD (2003). Role of the hypothalamic-pituitary axis in reinstatement of cocaine seeking behavior in squirrel monkeys. Psychopharmacology 168: 177-183.

Liu X, Vaupel DB, Grant S, London E (1998). Effect of cocainerelated environmental stimuli on the spontaneous electroencephalogram in polydrug abusers. Neuropsychopharmacology 19: $10-17$.

Lukas SE (1991). Topographic mapping during cocaine-induced intoxication and self-administration. In: Racagni G, Brunello N, Fukuda T (eds). Biological Psychiatry, Vol 2. Elsevier Science Publishers: New York. pp 25-29.

Lukas SE, Mendelson JH (1988). Electroencephalographic activity and plasma ACTH during ethanol-induced euphoria. Biol Psychiatry 23: 141-148.

Lukas SE, Mendelson JH, Amass L, Benedikt R (1990). Behavioral and EEG studies of acute cocaine administration: comparisons with morphine, amphetamine, pentobarbital, nicotine, ethanol and marijuana. NIDA Res Monogr 95: 146-151.

Lukas SE, Mendelson JH, Benedikt R (1995). Electroencephalographic correlates of marihuana-induced euphoria. Drug Alcohol Depend 37: 131-140.

Mattia D, Babiloni F, Romigi A, Cincotti F, Bianchi L, Sperli F et al (2003). Quantitative EEG and dynamic susceptibility contrast MRI in Alzheimer's disease: a correlative study. Clin Neurophysiol 114: 1210-1216.

McLellan AT, Luborsky L, Cacciola J, Griffith J, Evans F, Barr HL et al (1985). New data from the Addiction Severity Index: reliability and validity in three centers. J Nerv Ment Dis 173: 412-423.

Mendelson JH, Mello NK, Sholar MB, Siegel AJ, Kaufman MJ, Levin JM et al (1999). Cocaine pharmacokinetics in men and women during the follicular and luteal phases of the menstral cycle. Neuropsychopharmacology 21: 294-303.

Mezinskis J, Dyrenforth S, Goldsmith RJ, Somoza E (1998). Craving and withdrawal symptoms for various drugs of abuse. Psychiatr Ann 28: 577-583.

Noldy NE, Santos CV, Politzer N, Blair RDG, Carlen PL (1994). Quantitative EEG changes in cocaine withdrawal: evidence for long-term CNS effects. Pharmacoencephalography 30: 189-196.

Phillips RL, Herning R, London ED (1994). Morphine effects on the spontaneous electroencephalogram in polydrug abusers: correlations with subjective self-reports. Neuropsychopharmacology 10: $171-181$.

Porrino LJ, Lyons D, Miller MD, Smith HR, Friedman DP, Daunais JB et al (2002). Metabolic mapping of the effects of cocaine during the initial phases of self-administration in the nonhuman primate. J Neurosci 22: 7687-7694. 
Prichep LS, Alper KR, Kowalik SC, John ER, Merkin HA, Tom $\mathrm{M}$ et al (1996a). Quantitative electroencephalographic characteristics of crack cocaine dependence. Biol Psychiatry 40: $986-993$.

Prichep LS, Alper KR, Kowalik SC, Rosenthal MS (1996b). Neurometric qEEG studies of crack cocaine dependence and treatment outcome. J Addict Dis 15: 39-53.

Reid MS, Prichep L, O'Leary S, Ciplet D, Tom ML, Howard B et al (2003). Quantitative electroencephalographic studies of cueinduced cocaine craving. Clin EEG 34: 110-123.

Reid MS, Rose JE, Hart C, Foltin RW, Jones R (2004). Pharmacology alone does not a self-administered drug make: clinical laboratory studies on drugs of abuse. NIDA Res Monogr 184-Problems of Drug Dependence 2003: Proceedings of the 65th Annual Scientific Meeting, pp 126-134.

Rodriguez G, Nobili F, Copello F, Vitali P, Gianelli MV, Taddei G et al (1999). 99mTc-HMHPAO regional cerebral blood flow and quantitative electroencephalography in Alzheimer's disease: a correlative study. J Nucl Med 40: 522-529.

Roemer RA, Cornwall A, Dewart D, Jackson P, Ercegovac DV (1995). Quantitative electroencephalographic analysis in cocaine preferring polysubstance abusers during abstinence. Psychiat Res 58: 247-257.

Schutter DJ, van Honk J, de Haan EH, van Huffelen AC, Koppeschaar HP (2004). Cortisol, depression and reduced cortico-cortical cross-talk in Cushing's syndrome. J Endocrinol Invest 27: 683-686.

Sinha R, Talih M, Malison R, Cooney N, Andersen GM, Kreek MJ (2003). Hypothalamic-pituitary-adrenal axis and sympathoadreno-medullary responses during stress-induced and drug cueinduced cocaine craving states. Psychopharmacology 170: 62-72.
Stahl D, Ferger B, Kuschinsky K (1997). Sensitization to damphetamine after its repeated administration: evidence in EEG and behaviour. Naunyn-Schmeideberg's Arch Pharmacol 356: 335-340.

Steriade M, Gloor P, Llinas RR, Lopes da Silva FH, Mesulam M-M (1990). Basic mechanisms of cerebral rhythmic activities. Electroencephalogr Clin Neurophysiol 76: 481-508.

Steriade M, McCormick DA, Sejnowski TJ (1993). Thalamocortical oscillations in the sleeping and aroused brain. Science 262: 679-685.

Tolonen U, Sulg IA (1981). Comparison of quantitative EEG parameters from four different analysis techniques in evaluation of relationship between EEG and CBF in brain infarction. Electroencephalogr Clin Neurophysiol 51: 177-185.

Wallace EA, Wisniewski G, Zubal G, Van Dyck CH, Pfau SE, Smith EO et al (1996). Acute cocaine effects on absolute cerebral blood flow. Psychopharmacology 128: 17-20.

Ward AS, Collins ED, Haney M, Foltin RW, Fischman MW (1999). Blockade of cocaine-induced increases in adrenocorticotrophic hormone and cortisol does not attenuate the subjective effects of smoked cocaine in humans. Behav Pharmacol 10: 523-529.

Ward AS, Haney M, Fischman MW, Foltin RW (1997). Binge cocaine self-administration by humans: smoked cocaine. Behav Pharmacol 8: 736-744.

Yabase M, Carino MA, Horita A (1990). Cocaine produced cholinergically mediated analeptic and EEG arousal effects in rabbits and rats. Pharmacol Biochem Behav 37: 375-377.

Yoshida K, Gjerde DK, Carino MA, Halpern LM, Horita A (1993). The effect of SCH 23390 and raclopride on cocaine-induced analepsis and EEG arousal in rabbits. Neuropharmacology 32: 487-492. 